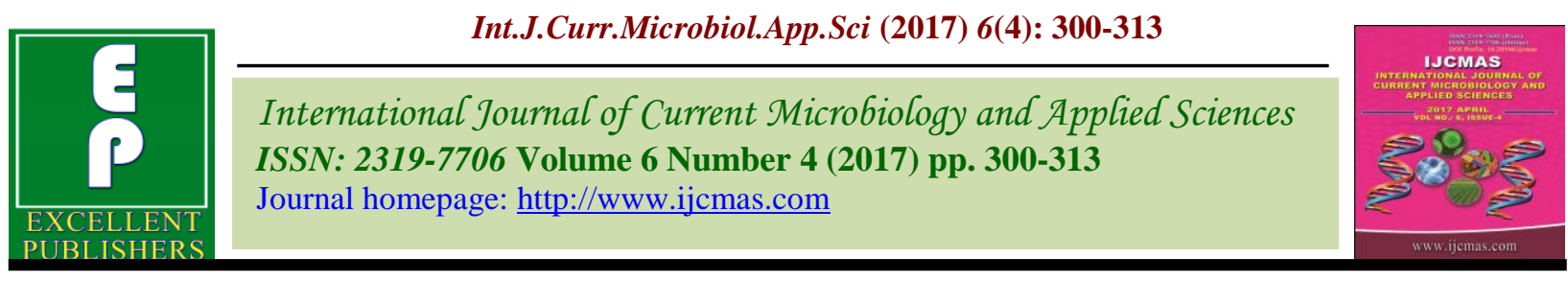

Review Article

https://doi.org/10.20546/ijcmas.2017.604.033

\title{
Role of Plant Volatiles in Defense and Communication
}

\author{
Rahul Kumar Meena ${ }^{1}$, Sumit Jangra ${ }^{1}$, Zeenat Wadhwa ${ }^{1}$, \\ Monika ${ }^{2}$ and Leela Wati ${ }^{2}$ \\ ${ }^{1}$ Department of Molecular Biology, Biotechnology and Bioinformatics, \\ CCS Haryana Agricultural University, Hisar, India \\ ${ }^{2}$ Department of Microbiology, CCS Haryana Agricultural University, Hisar, India \\ *Corresponding author
}

\section{A B S T R A C T}

\section{Keywords}

Plant volatiles,

Signal transduction,

Defense, Ecological

functions.

\section{Article Info}

Accepted:

02 March 2017

Available Online:

10 April 2017
Plant releases volatile chemicals compounds in air through which plant interacts to both biotic and abiotic communities these are called plant volatiles. Nearly one-fifth of the carbon fixed by plant every day is released as volatiles. Majority of plant volatiles are derived from: terpenoids, fatty acid catabolites, aromatics, amino-acid derived products and alcohols. Plant volatiles serve various functions in plants ranging from signal transduction to plant defense. Volatiles compounds released by plants are one of the fastest exploited weapons, with respect to minimizing the yield losses and providing tolerance to various stresses. Plant volatiles have several physiological and ecological functions for plants. Many of the volatile terpenes are nonspecific toxins active against a wide range of organisms (bacteria, fungi, plants and animals). Agricultural crops are having less volatile emission making them susceptible to various biotic and abiotic stress. Keeping in mind the ecological functions of volatiles, the aim should be to optimize the emission patterns by choosing right cultivars for individual land areas with specific cultivation challenges. Plant volatiles are also a source of intra plant and inter plant interactions. In response to attacks, plants produce a wide array of volatile organic compounds which have multiple functions as internal plant hormones as well as play a crucial role in the communication between plants and the associated community members, such as other herbivores, phytopathogens and the natural enemies of herbivores.

\section{Introduction}

Plant interacts with a wide range of biotic and abiotic communities both beneficial as well as deleterious. These biotic and abiotic communities induce a wide range of responses in plants, including physical and chemical defense responses. Nearly a million of insect species are found on plants and its surroundings, and nearly half of them feed on plants. This ongoing battle between plants and insects has lasted over 350 million years (Gatehouse, 2002). With increase in time insects have evolved to locate their host plant for feeding, oviposition and using plant exudates in various manners. Among these are a plethora of attackers, including herbivorous arthropods and plant pathogens. Half of the estimated 6 million insect species are herbivorous (Schoonhoven et al., 2005). To protects itself from the deleterious effects of insect's plants have evolved an expiate defense mechanism. They outfit themselves with forcible barriers, such as thorns, 
trichrome and cuticles. Plant secondary metabolites are powerful defense weapon against various insecticidal attacks. Nearly more than 500,000 secondary metabolites are found in plants (Mendelsohn and Balick, 1995). In spite of this there are other defense mechanism which includes glucosinolates, cyanogenic glucosides, alkaloids, phenolics, and proteinase inhibitors (PIs), function as toxins, repellents or antidigestives, these all are part of direct defense other than this there is indirect defense which includes green leaf volatiles, volatile organic compounds and extrafloral nectars which attract the natural enemies (such as parasitoids) of herbivores. These two powerful defense systems, acquired by plants during the long arms race with herbivores, have enabled plants to survive (Kessler and Baldwin, 2002).

\section{Plant volatiles}

Plant volatiles are the metabolites that plant releases in the air and can circulate at ambient temperature. Nearly one-fifth of the atmospheric $\mathrm{CO}_{2}$ fixed by plants is put back into the air as volatiles each day. Plants are genius synthetic chemists; they use their anabolic skills to synthesize volatiles, which protect plants against both biotic and abiotic stresses. Plant volatiles also known as Green leaf volatiles (GLVs) are semiochemicals used by insects to find their food or their conspecifics. Volatiles have been reported to be a key in indirect defenses and to have a direct effect on pests. They are probably one of the fastest exploited weapons and are also able to directly elicit or prime plant defense responses. Due to these reasons GLVs should be mooted as co-protagonists in the play between plants and their attackers. Plant volatiles are primarily released through the membranes of the epidermal tissues, where they are synthesized or from other structures such as trichomes, osmophores or crenulated epidermal cells commonly found in flower parts whereas in leaves and stems, volatiles are released from the stomatal pores through which $\mathrm{CO}_{2}$ is assimilated and water vapor is released. Some volatiles are also stored in conjugated forms in vacuoles or in specialized ducts and laticifers. There are volatiles which can freely pass cell membrane and be released into the atmosphere and root associated soil these are called non-conjugated volatiles. Few volatiles released from flowers and fruit provides chemical cues to pollinators and seed disseminators, thereby ensuring the plant's reproductive success.

\section{Diversity of plant volatiles}

The majority of plant volatiles are derived from four biosynthetic classes: terpenoids, fatty acid catabolites, aromatics, amino-acid derived products and alcohols of 6-carbon compounds released after wounding (Dudareva et al., 2006). Green leaf volatiles (E)-2-hexenal, (Z)-3-hexen-1-ol 35 and cis-3hexenyl acetate, terpenes myrcene and blended ocimene volatiles (E) ocimene, $(\mathrm{Z})$ ocimene and allo-ocimene and the phytohormones methyl jasmonate, 21 methyl salicylate and ethylene. Volatiles are produced via the lipoxygenase pathway where emitted rapidly upon disturbance of the plant, by mechanical damage as well as herbivore feeding. These compounds are therefore indicative of any mechanical damage and could provide early signals to receiving plants. Although these compounds are generally found in very small amounts relative to the total weight of the plant (the essential oil of rose petals comprises only $0.02-0.03 \%$ of their weight), as a group, they are of tremendous importance, both ecologically for the plants and aesthetically and commercially for human.

Terpenoids are the largest group of secondary compounds, consisting of approximately 40,000 compounds, including at least 1,000 
monoterpenes and 6,500 sesquiterpenes. All terpenoids originate from isopentenyl diphosphate (IPP) and its allylic isomer dimethylallyl diphosphate (DMAPP), which are derived via two alternative pathways. In the cytosol, IPP is synthesized via the mevalonic acid (MVA) pathway, while in plastids it is synthesised via the 2-C-methylD-erythritol 4-phosphate (MEP) pathway (Arimura et al., 2004). Some terpenoids are constituents of essential oils and resins and are constitutively produced and stored in specialised structures such as glandular trichomes or resin ducts. Upon damage by herbivores these structures are broken and the compounds are released. The de novo biosynthesis of terpenoids can be induced locally and systemically by herbivore feeding. Terpenoids as a group are therefore, able to provide rapid, but also herbivore-damage related signals to receiving plants.

Methyl jasmonate is a volatile derivative of jasmonic acid, which is an integral component of plant defence responses to insect feeding. Methyl salicylate is synthesized from salicylic acid, it is a phenolic compound and play an important role in plant defense in response to aphid feeding damage and is emitted by tobacco in response to tobacco mosaic virus infection. Tobacco plants exposed to methyl salicylate have been shown to increased resistance to tobacco mosaic virus.

Among the one- and two-carbon plant volatiles included in the volatile blends, methanol and ethylene are most commonly emitted. Methanol is released in part from the demethylation of the abundant cell-wall constituent pectin when leaves change shape during growth or senescence, or when they are attacked by herbivores having high $\mathrm{pH}$ oral secretions. The shift in $\mathrm{pH}$ at the feeding site activates pectin methyl esterases in the cell wall, releasing methanol, frequently in amounts ten times higher than the next most abundant group of volatiles emitted from damaged leaves. Ethylene is among the plant hormones that are emitted into the air in biologically active form and is derived by the oxidation of 1-amino-cyclopropane-1carboxylicacid, which in turn is derived from the amino acid methionine.

\section{Biosynthesis of plant volatiles}

Volatiles production is the primary or sole way through which plants cross talk with environment. Different metabolic pathways put up to the volatiles that are released, and hence the volatile metabolome contains information about the plant's metabolic status. The biosynthesis of volatiles occurs in the epidermal cells of plant tissues or in specialized surface structures such as trichomes. In basil, peltate glandular trichomes on the leaf surfaces have been shown to serve as highly active metabolic factories producing and storing large amounts of phenylpropanoid-derived plant volatiles. In general, metabolic pathways across several cellular compartments are integrated in biosynthesis of plant volatiles. In some cases, volatiles are subjected to glycosylation and stored in vacuoles. Some nonvolatile precursors, such as glucosinolates and cyanogenic glycosides, are stored in vacuoles as well and are enzymatically hydrolyzed when plant tissues containing these substances are disrupted, releasing volatile degradation products.

Discovery of many transcription factors that specifically coordinate the many steps involved in plant volatile biosynthesis. Coordination of biosynthesis and release of plant volatiles is regulated by signaling pathways that control the production of chemical defenses, toxins and digestibility reducing compounds in response to attack from insects and pathogens also. The volatiles that are released after herbivore attack is 
regulated by oxylipin signaling pathway, which plays a central role in normalizing many of these inducible defenses.

\section{Herbivore induced plant volatiles}

\section{Multifunctional weapon against herbivores}

Plants under herbivore attack synthesize defensive organic compounds that directly or indirectly affect herbivore performance and mediate other interactions with the community. The so-called herbivore induced plant volatiles (HIPVs) consist of odors released by attacked plants that serve as important cues for parasitoids and predators to locate their host/prey. Herbivore-induced plant volatiles (HIPV) can mediate indirect defenses i.e. by attracting foraging carnivorous predators and parasitoids that kill herbivores (Dicke et al., 2009; Mumm et al., 2010). Dicke and Sabelis (1988) were the first to show that HIPVs indeed can be key foraging cues for natural enemies of herbivores. As GLVs are immediately released from the wounded leaf of a plant, this group of volatiles can provide rapid and reliable information about the exact location of the attacking herbivore. However, because GLVs are released from almost every plant and under various stress conditions, they might not provide reliable information to the prey-searching carnivore. Plants ready their defenses in response to a signal or previous challenge so that they can respond with increased rigour should they be subsequently challenged by herbivores or pathogens also termed as priming. The priming of plants as a product of plant-plant communication via volatile organic compounds is a recent discovery. Volatiles emitted by herbivoredamaged plants are complex blends basically made of green leaf volatiles. The release of these compounds generally follows a temporal pattern, being GLVs emitted first since they are released from damaged cell membranes (Hatanaka et al., 1987) and the others volatiles, which are de novo synthesized and emitted latter (Paré and Tumlinson, 1997; Turlings et al., 1998a). Other plant organs besides photosynthetic plant tissues also release HIPVs, which is the case of plant roots (Rasmann et al., 2005). Volatiles released by herbivory are important signal that improve the fitness of the plant by eliciting behavioral responses in herbivore natural enemies and thus increasing the predation rate leading to reduced plant damage. This plant response has often been referred to as a 'cry for help', due to natural enemies of herbivores using these volatile signals as cues in the process of prey or host foraging. The connotation behind "cry for help" was that plants release odor blends signaling to specific natural enemies in order to help in their defense against herbivore attack. These compounds are emitted by plants in different quantities and ratios depending on the herbivore, which determines the attractiveness of the emitted volatile blend to different species of foraging predators. The composition of emission is often plant specific and herbivory specific to the species level and even to the level of larval feeding stage. Plant induced response is triggered by a combination of cell damage (Heil, 2009) and contact with elicitors, two main groups: fatty acid-amino acid conjugates and lytic enzymes present in the herbivore oral secretions (Mattiacci et al., 1995; Halitschke et al., 2001 and Truitt et al., 2004), which activate signaling pathways (lipoxygenase, shikimate, and isoprenoid) coordinated by three main plant hormones: jasmonic acid (JA), salicylic acid (SA) and ethylene (Walling, 2000).

VOCs induced by herbivory attract natural enemies of herbivores, which may confer protection to the plant (Kessler and Baldwin 2001; Dicke and Baldwin 2010). Induced volatiles can have repellent effects on herbivores (Delphia, Mescher and De Moraes 
2007; Bleeker et al., 2009) and inhibit pathogen colonization (Brown et al., 1995). In addition, they are involved in information transfer between and within plants, through the airborne transport of signals that lead to the priming or expression of defences in neighbouring plants or in distal parts of the emitting plant (Engelberth et al., 2004; Kessler et al., 2006; Frost et al., 2007). These VOCs, however can be a double-edged sword as they can also be used by herbivores as host-plant location cues (Bolter et al., 1997). Plant volatiles emitted by herbivore infestation can inactivate pathogens and vice versa. Herbivory can lead to the activation of pathogenesis related compounds, but they do not affect the herbivores. Herbivore-damaged plants also release MeSA, which is thought to prepare neighboring plants against pathogenic microbes (Kessler and Baldwin, 2001; Agrawal et al., 2002; James, 2003; Kant et al., 2004).

The timing of the deployment also defines defenses. Constitutive defenses are physical and chemical defensive traits that plants have regardless of the presence of herbivores; in contrast, inducible defenses are mounted only after plants are attacked by herbivores. Therefore, inducible defenses are particularly interesting, since they endow plants with flexible and economy-friendly defense systems. Further, two types of induced plant defence are distinguished: (a) direct defence that affects the performance or behaviour of its attacker directly, e.g. through an increased concentration of secondary metabolites (Gols et al., 2008; Steppuhn et al., 2004), including plant volatiles (De Moraes et al., 2001; Kessler and Baldwin, 2001). It was reported to occur in glandular trichomes (Schilmiller $e t$ $a l ., 2008)$, which are involved in the synthesis and storage of a large array of specialized metabolites such as terpenoids and phenylpropanoids (Wink, 2003; Gang, 2005; Naoumkina et al., 2010), proteinase inhibitors
(Liu et al., 2006) and polyphenol oxidase (Yu et al., 1992). The stored toxic terpenoid volatiles are only released from the glandular trichomes to directly repel herbivores, and thus are classified as constitutive direct defenses. Herbivory induces defense responses not only in the wounded regions but also in undamaged regions in the attacked leaves and in distal intact (systemic) leaves. (b) Indirect defence that enhances the effectiveness of natural enemies of herbivores through the production of HIPV (Dicke and Baldwin, 2010), and through the induction of extra floral nectar (EFN) (Dicke, 2009; Heil, 2008). The induced production of volatile organic compounds (VOCs) that attract carnivorous arthropods can occur in response to herbivore feeding damage (Vet and Dicke, 1992) or egg deposition (Hilker and Meiners, 2002), both aboveground (Arimura et al., 2005) and belowground (Erb et al., 2009a). Defense is costly; thus discerning herbivory from casual mechanical wounding and promptly deploying increased levels of defensive compounds are critical skills in the battle between plants and herbivores. Various microbial (or pathogen) associated molecular patterns (MAMPs or PAMPs) are recognized by specific receptors. The $\mathrm{R}$ gene, mediated defense system detects the presence of Avr proteins secreted from pathogens and initiates the hypersensitive response. Similarly, herbivore derived elicitors or cues function as herbivore associated molecular patterns (HAMPs) (Mithofer and Boland, 2008). These HAMPs may function in concert with herbivory-induced molecules originated from plants themselves to elicit the full patina of defense responses (Heil, 2009).

\section{HI-VOCs as promising tools in biocontrol}

HI-VOCs appear to be a promising tool for biocontrol and several scientists have attempted to transform plants to enhance their potential to emit HI-VOCs. However, the 
only system in which plant odours are consciously and successfully being used for biocontrol in agriculture appears to be the push-pull system (Khan et al., 1997; Hassanali et al., 2008). For example, commercial cultivars of cotton (Gossypium hirsutum) release seven times lower overall quantities HI-VOCs than a naturalized line (Loughrin et al., 1995) and North American cultivars of maize do not emit the nematode attractant (E)-b caryophyllene from their roots (Rasmann et al., 2005). It is unlikely that HIVOCs have been consciously counter selected in plant breeding and, indeed, the quantities of emitted VOCs have not been reduced in certain Brassica and Phaseolus cultivars (Benrey et al., 1998) or in the abovegroundparts of several maize lines (Gouinguen et al., 2001). However, it appears likely that many crops suffer from reduced capacities to attract and maintain beneficial arthropods.

\section{Biological functions of plant volatiles}

Plant volatiles have several physiological and ecological functions for plants. Many of the volatile terpenes are nonspecific toxins active against a wide range of organisms (bacteria, fungi, plants and animals). Certain monoterpenes have been found to inhibit the growth of competing plants (a process called allelopathy), and many have been found to be toxic to plant pathogens and insects. Many other volatile terpenes appear to act as feeding deterrents to herbivores. They are mostly localized and present in higher concentrations in buds, leaves and juvenile tissues than in mature plant tissues most likely to minimize grazing of young plant parts. Volatile compounds are also connected to several plant physiological functions. Especially isoprene is well known for its thermo protective function on photosynthetic processes (Behnke et al., 2007; Loreto and Schnitzler, 2010) and moreover, the compound was shown to (directly or indirectly) quench reactive oxidative species (ROS) in plant tissue (Loreto and Velikova, 2001).

Floral scent bouquets may contain from one to 100 volatiles, but most species emit between 20 and 60 different compounds. The total amount of emitted floral volatiles varies from the low picogram range to more than $30 \mu \mathrm{g} / \mathrm{h}$ with the largest amounts produced by flowers of various beetle and moth pollinated species (Knudsen and Gershenzon, 2006). Therefore, to attract pollinators and seed disseminators and thus to ensure reproductive and evolutionary success, many of these flowering species release diverse blends of volatile compounds from their flowers and fruits in addition to visual and tactile cues (Buchmann and Nabhan, 1996; Dudareva and Pichersky, 2000). Many floral volatiles have antimicrobial or antifungal activity (DeMoraes et al., 2001; Friedman et al., 2002; Hammer et al., 2003) and could also act to protect valuable reproductive plant organs from pathogens. Volatiles involved in antimicrobial defense are often produced in pistils and/or nectaries, as was shown for linalool and linalool oxide in flowers of Clarkia species (Pichersky et al., 1994; Dudareva et al., 1996) and for sesquiterpene and monoterpene formation in Arabidopsis flowers (Chen et al., 2003; Tholl et al., 2005).

Volatile compounds emitted from fruits determine the overall aroma properties and taste, and thus could play a role in the attraction of animal seed dispersers. Other research suggests that odours can influence our behaviour and health, in what has been termed 'aroma therapy' (Dorschner, 1995). Pleasant-smelling plant odours such as lavender, lemon and jasmine have been found to reduce blood pressure and boost productivity among office workers, although foul-smelling compounds have been found to lead to higher levels of depression. Several 
volatiles are utilised for their therapeutic effects. 1,8-Cineole, the major constituent of eucalyptus (Eucalyptus globulus) and rosemary (Rosmarinus officinalis) oils, acts as a general stimulant and improves locomotive function in humans. Eugenol, the main constituent of clove (Eugenia caryophyllus) oil, is still used to treat toothache owing to its analgesic properties. Another use of plant volatiles is as food additives. During processing, many odour volatiles are lost, but replacing these so that the original flavour of the food is matched may be extremely difficult and one of the most familiar uses of plant volatiles by humans is as perfumes.

Plants also release volatiles from their roots with chemical and structural diversity comparable to those found in emissions from aerial plant organs. Similar to aboveground volatile compounds, root volatiles can contribute to a belowground defense system by acting as antimicrobial or antiherbivore substances, or by attracting enemies of root feeding herbivores. Involvement of plant volatiles in defense and reproductive processes, volatile isoprenoids are able to protect plants from heat damage and allow them to maintain photosynthetic rates thus enhancing plant thermotolerance at elevated temperatures (Sharkey et al., 2001; Loreto et al., 1998; Copolovici et al., 2005; Penuelas et al., 2005; reviewed in Sharkey and Yeh, 2001). Volatile isoprenoids may also serve as antioxidants, protecting plants against a wide range of stresses including ozone-induced oxidative stress (Loreto et al., 2001; Loreto and Velikova, 2001) and singlet oxygen accumulation (Affek and Yakir, 2002). Although the mechanism of isoprene protection of plants against oxidative stress still remains unclear, it has been shown that isoprene may have direct ozone quenching property rather than inducing resistance at the membrane level (Loreto et al., 2001). The herbivory defenses of plants may be expressed constitutively or they may be induced and developed only after attack. This is a question of benefit versus cost, since plant defense mechanisms are expensive. Plants are constantly in the dilemma of combining growth and development with defense. Volatiles released from herbivore infested plants also mediate plant-plant interactions and may induce the expression of defense genes and emission of volatiles in healthy leaves on the same plant or of neighboring unattacked plants, thus increasing their attractiveness to carnivores and decreasing their susceptibility to the damaging herbivores (Dicke et al., 1990; Arimura et al., 2002, 2004b; Ruther and Kleier, 2005). Volatiles can directly affect herbivores' physiology and behavior due to their toxic, repelling, or deterring properties (Bernasconi et al., 1998; De Moraes et al., 2001; Kessler and Baldwin, 2001; Vancanneyt et al., 2001; Aharoni et al., 2003). They can also attract enemies of attacking herbivores, such as parasitic wasps, flies or predatory mites, which can protect the signaling plant from further damage (Dicke et al., 1990; Turlings et al., 1990; Vet and Dicke, 1992; Pare and Tumlinson, 1997; Drukker et al., 2000; Kessler and Baldwin, 2001). Moreover, some volatile compounds can mediate both direct and indirect defenses, deterring lepidopteran oviposition and attracting herbivore enemies as was found in Nicotiana attenuata (Kessler and Baldwin, 2001).

\section{Plant volatiles and smart agriculture}

Agricultural crops (i.e. maize, wheat and rice) typically have low volatile emissions, but many woody species cultivated for biomass production such as oil palm, poplar, willow and eucalyptus emit higher levels of volatiles than the natural vegetation. As volatile emissions from different species and cultivars can vary greatly, the choice of cultivars is an easy starting point to manage volatile release 
into the atmosphere and hence their air quality impacts. However, volatile emissions are not only something to avoid. Keeping in mind the ecological functions of volatiles, the aim should be to optimize the emission patterns by choosing right cultivars for individual land areas with specific cultivation challenges. Thus, the potentially negative atmospheric effects could be reduced while the plant yield and stress tolerance are enhanced through optimal volatile pattern. Modern biological and chemical techniques (e.g. natural chemical stimulants, such as jasmonates) can be used to optimize the volatile patterns to prime the plants and to trigger plant-plant or plant-insect communications for biological pest and weed control (Rosenkranz et al., 2014). There is desired to be developed optimal cultivars for different agricultural systems and climates, plant phenotyping tools that include volatile emission analysis. Volatile formation in plants via metabolic engineering to improve scent and aroma quality of flowers and fruits or to enhance crop protection through direct and indirect plant defense (L"ucker et al., 2006; Dudareva and Pichersky, 2006; Degenhardt et al., 2003; Aharoni et al., 2005), in general, the bioengineering of volatiles can be achieved either through the modification of existing pathways or by the introduction of new gene(s) normally not found in the host plant. Improvement of volatile-based direct plant defense was accomplished by over expressing a dual linalool/nerolidol synthase (FaNES1) from strawberry in Arabidopsis chloroplasts. Linalool and its derivatives produced by the transgenic plants significantly repelled an agricultural pest, the aphid Mysus persicae, in dual-choice assays (Aharoni et al., 2003).

\section{Tritrophic interactions: plant communication}

The volatiles emitted from vegetative tissues as a part of the plant defense system can directly repel microbes and animals or attract the natural predators of attacking herbivores, which indirectly protects the plant via tritrophic interactions. Volatile compounds also play a major role in plant communication and indirect defence. In tritrophic communication plants attract herbivore enemies i.e. calls for 'bodyguards' to localize their hosts. Such indirect defence provides plants a top-down control of herbivore populations that was for the first time observed within spider mite-infested Lima beans calling carnivorous mites for help (Dicke and Sabelis, 1988) and was later shown to be a more general phenomenon between several plants and predator or parasitoid species. Volatiles are used as well in plant-plant communication so that plants under herbivore attack can alert neighbouring plant species, priming their chemical defenses (Heil, 2014). The volatile phytohormones methyl salicylate and methyl jasmonate serve as important signaling molecules for communication purposes, and interact with each other to optimize the plant defense response.

\section{Plant communication}

In nature, plants are often confronted with simultaneous or sequential attack, yet even until recently research was largely conducted on study systems comprising of single plantattacker combinations (Dicke, van Loon and Soler 2009). In response to attacks, plants produce a wide array of volatile organic compounds (VOCs) which have multiple functions as internal plant hormones (ethylene, methyl jasmonate, jasmonic acid) as well as play a crucial role in the communication between plants and the associated community members, such as other herbivores, phytopathogens and the natural enemies of herbivores. Plant communication was first reported in 1983. This includes interspecific communication, intraspecific communication and within plant communication. The term 'talking trees' or 
'plant-plant communication' was coined to describe VOC mediated interactions of plants with their neighbours, whereas the attraction of carnivores to prey on herbivores was termed 'indirect defence', the compounds can attract the natural predators of pathogens and herbivores through an indirect defense mechanism. Terpenoids and GLV compounds emitted from vegetative or floral tissues can directly repel herbivores or inhibit the spread of plant pathogens via antibacterial and antifungal activities. Terpenoids and GLVs are plant volatiles which involved in plantplant communication. Since metabolites of JA and SA, such as MeSA or MeJA, can cross the cell membrane and are volatile in nature, they are thought to be important signaling compounds for intra-plant and inter-plant communication. Plants communicate with neighboring plants, insects (herbivore and carnivore) and microbes, including pathogens, through plant volatiles that are released due to herbivory or pathogen attack. Plants emit volatile compounds that can act as a communication method to insects, neighboring plants and pathogens. Plants respond to leaf and root damage by herbivores and pathogens by emitting these compounds. The volatile compounds can deter the herbivores or pathogens directly or indirectly by attracting their natural enemies to kill them.

The simultaneous damage of plants by herbivores and pathogens can influence plant defense. Receivers of herbivore induced volatile organic compounds (HI-VOCs) comprise distant parts of the same plant (within-plant signaling), neighbouring plants (plant-plant signaling), herbivores, and multiple carnivores that respond to the 'plant's cry for help', such as parasitoids and hyperparasitoids, entomopathogenic nematodes, and predatory mites, beetles, bugs and birds. Large intraspecific variations in herbivory-induced signaling events and secondary metabolites exist among different plant populations and even individuals within a population.

\section{Signal transduction pathways regulating induced plant defences}

Herbivore attack induces a battery of molecular events in plant cells, which transduce the alarm signals and eventually result in the accumulation of defensive metabolites. Although little is known about how plants perceive herbivory, many small molecules, as well as proteins, have been identified to be the nodes of complex regulatory networks that enable plants to optimize energy and resource allocation and deploy appropriate defenses. Herbivory induced changes are mediated by elaborate signaling networks, which include receptors/sensors, $\mathrm{Ca}^{2+}$ influxes, kinase cascades, reactive oxygen species, and phytohormone signaling pathways. Plants sense the existence of herbivores and initiate changes in a battery of signaling pathways, including $\mathrm{Ca}^{2+}$ influxes, membrane depolarization, kinase activation, and jasmonate accumulation. These pathways form sophisticated intertwined regulatory networks that orchestrate specific defense responses according to the species of the herbivore. At least three phytohormones, that is, salicylic acid (SA), jasmonic acid (JA) and ethylene (ET), play key regulatory roles in the interconnecting signal-transduction pathways that mediate induced defences in response to herbivore and pathogen infestation. A shortdistance mobile signal travels from damaged regions in an herbivore-attacked leaf to certain regions of the undamaged portion and initiates defense reactions; moreover, a longdistance mobile signal conveys an herbivory alert to distal intact leaves and subsequently triggers defenses in these systemic leaves. Although different plant species may have different mechanisms with which to activate 
systemic responses, the jasmonate pathway is required for systemic responses. Rapid advances in our knowledge on the underlying mechanisms of plant defenses have shown that the interactions under a multiple attack scenario are complex (Pieterse et al., 2012).

In conclusion extensive progress has been made in the past decade in the field of plant volatiles which is depicted by the information available on the biology, ecology and biochemistry of plant volatiles. Little is known about the role volatiles of signaling cascade involved in plant-plant communication and plant-insect interactions, which is waiting for future investigation. Development of new model systems for experimentation is a major challenge. Recently the use of transcriptomics and metabolomics in plant volatile research has revolutionized the understanding of regulatory properties of pathways involved in volatiles formation and signaling.

New insights in the study of plant communication through volatiles will be provided by the comparative study of transcriptomic, proteomic, and metabolomic datasets of both emitters (plants) and receivers (plants, insects/animals). Study of transcription factors involved in emission of volatiles will led to understanding of intracellular metabolite trafficking and the mechanism of the release process, which will significantly lead to metabolic engineering of plant volatiles pathways for betterment of agricultural crops. Further the use of gene knock down and transgenic technologies will allow us to determine the key compounds involved in plant-insect and plant-plant interactions. The knowledge from above studies could be used to develop crop plants with desired/improved agronomic traits such as pest and disease resistance, weed control, improved fragrance of ornamentals and pollination of seed crops, enhanced aroma of fruits and vegetables, and the production of pharmaceuticals in plants.

\section{References}

Affek, H.P., and Yakir, D. 2002. Protection by isoprene against singlet oxygen in leaves. Plant Physiol. 129: 269-277.

Agrawal, A.A., Janssen, A., Bruin, J., Posthumus, M.A., and Sabelis, M.W. 2002. An ecological cost of plant defence: attractiveness of bitter cucumber plants to natural enemies of herbivores. Ecol. Lett., 5: 377-385.

Aharoni, A., Giri, A.P., Deuerlein, S., Griepink, F., de Kogel, W.J., Verstappen, F.W.A., Verhoeven, H.A., Jongsma, M.A., Schwab, W. and Bouwmeester, H. J. 2003. Terpenoid metabolism in wild-type and transgenic Arabidopsis plants. Plant Cell, 15: 2866-2884.

Aharoni, A., Jongsma, M.A., and Bouwmeester, H.J. 2005. Volatile science? Metabolic engineering of terpenoids in plants. Trends Plant Sci., 10: 594-602.

Arimura, G., Huber, D.P.W. and Bohlmann, J. 2004a. Forest tent caterpillars (Malacosoma disstria) induce local and systemic diurnal emissions of terpenoid volatiles in hybrid poplar (Populus trichocarpa $\times$ deltoides): cDNA cloning, functional characterization, and patterns of gene expression of (-) germacrene D synthase, PtdTPS1. Plant J., 37: 603-616.

Arimura, G., Kost, C. and Boland, W. 2005. Herbivore-induced, indirect plant defences. Biochim. Biophys. Acta., 1734: 91-111.

Arimura, G., Ozawa, R., Nishioka, T., Boland, W., Koch, T., Kuhnemann, F. and Takabayashi, J. 2002. Herbivore-induced volatiles induce the emission of ethylene in neighboring lima bean plants. Plant J., 29: 87-98.

Arimura, G., Ozawa, R., Kugimiya, S., Takabayashi, J., and Bohlmann, J. 2004b. Herbivore-induced defense response in a model legume: Two-spotted spider mites, 
Tetranychus urticae, induce emission of (E)- $\beta$-ocimene and transcript accumulation of (E)- $\beta$-ocimene synthase in Lotus japonicus. Plant Physiol., 135: 1976-1983.

Baldwin, I.T., Kessler, A. and Halitschke, R. 2002. Volatile signaling in plant plantherbivore interactions: What is real? Curr. Opin. Plant Biol., 5: 351-354.

Behnke, K., Ehlting, B. and Teuber, M. 2007. Transgenic, non-isoprene-emitting poplars don't like it hot. Plant J., 51: 485-499.

Benrey, B., Callejas, A., Rios, L., Oyama, K. and Denno, R.F. 1998. The effects of domestication of Brassica and Phaseolus on the interaction between phytophagous insects and parasitoids. Biol. Control, 11: 130-140.

Bernasconi, M.L., Turlings, T.C.J., Ambrosetti, L., Bassetti, P. and Dorn, S. 1998. Herbivore-induced emissions of maize volatiles repel the corn leaf aphid, Rhopalosiphum maidis. Entomol. Exp. Appl., 87: 133-142.

Bolter, C.J., Dicke, M., Van Loon, J.J., Visser, J.H. and Posthumus, M.A. 1997. Attraction of Colorado potato beetle to herbivore-damaged plants during herbivory and after its termination. $J$. Chem. Ecol., 23: 1003-1023.

Buchmann, S.L. and Nabhan, G.P. 1996. The Forgotten Pollinators. Island Press, Washington D.C.

Chen, F., D'Auria, J.C., Tholl, D., Ross, J. R., Gershenzon, J., Noel, J. P. and Pichersky, E. 2003. An Arabidopsis thaliana gene for methylsalicylate biosynthesis, identified by a biochemical genomics approach, has a role in defense. Plant J., 36: 577-588.

Copolovici, L.O., Filella, I., Llusia, J., Niinemets, U. and Penuelas, J. 2005. The capacity for thermal protection of photosynthetic electron transport varies for different monoterpenes in Quercus ilex. Plant Physiol., 139: 485-496.

De Moraes, C.M., Mescher, M.C., and Tumlinson, J.H. 2001. Caterpillar induced nocturnal plant volatiles repel conspecific females. Nature, 410: 577-580.

Degenhardt, J., Gershenzon, J., Baldwin, I.T., and Kessler, A. 2003. Attracting friends to feast on foes: Engineering terpene emission to make crop plants more attractive to herbivore enemies. Curr. Opin. Biotechnol., 14: 169-176.

Delphia, C., Mescher, M. and De Moraes, C. 2007. Induction of plant volatiles by herbivores with different feeding habits and the effects of induced defenses on host-plant selection by thrips. J. Chem. Ecol., 33: 997-1012.

Dicke, M. and Sabelis, M.W. 1988. How plants obtain predatory mites as bodyguards. Netherlands J. Zool., 38: 148-165.

Dicke, M. 2009. Behavioural and community ecology of plants that cry for help. Plant Cell Environ., 32: 654-665.

Dicke, M., and Baldwin, I.T. 2010. The evolutionary context for herbivoreinduced plant volatiles: beyond the 'cry for help'. Trends Plant Sci., 15: 167-175.

Dicke, M., Sabelis, M.W., Takabayashi, J., Bruin, J., and Posthumus, M.A. 1990. Plant strategies of manipulating predatorprey interactions through allelochemicals: prospects for application in pest control. J. Chem. Ecol., 16: 3091-3118.

Dicke, M., Van Beek, T.A., Posthumus, M.A., Ben Dom, N., Van Bokhoven, H. and De Groot, A. 1990. Isolation and identification of volatile kairomone that affects acarine predator prey interactions Involvement of host plant in its production. J. Chem. Ecol., 16: 381-396.

Dicke, M., Van Loon, J.J.A., and Soler, R. 2009. Chemical complexity of volatiles from plants induced by multiple attack. Nat. Chem. Biol., 5: 317-324.

Drukker, B., Bruin, J., Jacobs, G., Kroon, A., and Sabelis, M.W. 2000. How predatory mites learn to cope with variability in volatile plant signals in the environment of their herbivorous prey. Exp. Appl. Acarol., 24: 881-895.

Dudareva, N., and Pichersky, E. 2000. Biochemical and molecular genetic aspects of floral scents. Plant Physiol., 
122: 627-633.

Dudareva, N., and Pichersky, E. 2006. Metabloic engineering of floral scent of ornamentals. J. Crop. Improv., in press.

Dudareva, N., Cseke, L., Blanc, V.M., and Pichersky, E. 1996. Evolution of floral scent in Clarkia: Novel patterns of Slinalool synthase gene expression in the C. breweri flower. Plant Cell, 8: 11371148.

Dudareva, N., Negre, F., and Nagegowda, D.A. 2006. Plant volatiles: recent advances and future perspectives. Crit. Rev. Plant Sci., 25: 417-440.

Engelberth, J., Alborn, H.T., Schmelz, E.A., and Tumlinson, J.H. 2004. Airborne signals prime plants against insect herbivore attack. Proc. Natl. Acad. Sci., USA, 101: 1781-1785.

Erb, M., Flors, V., Karlen, D., de Lange, E., Planchamp, C., D'Alessandro, M., Turlings, T.C.J., and Ton, J., 2009a. Signal signature of aboveground-induced resistance upon belowground herbivory in maize. Plant J., 59: 292-302.

Friedman, M., Henika, P. R., and Mandrell, R. E. 2002. Bactericidal activities of plant essential oils and some of their isolated constituents against Campylobacter jejuni, Escherichia coli, Listeria monocytogenes, and Salmonella enterica. J. Food Protect., 65: 1545-1560.

Frost, C., Appel, H., Carlson, J., De Moraes, C., Mescher, M., and Schultz, J. 2007. Within-plant signalling by volatiles overcomes vascular constraints on systemic signalling and primes responses against herbivores. Ecol. Lett., 10: 490498.

Gang, D.R. 2005. Evolution of flavors and scents. Annu. Rev. Plant Biol., 56: 301325 .

Gatehouse, J.A. 2002. Plant resistance towards insect herbivores: a dynamic interaction. New Phytol., 156: 145-69.

Gols, R., Bukovinszky, T., van Dam, N.M., Dicke, M., Bullock, J.M., and Harvey, J.A., 2008. Performance of generalist and specialist herbivores and their endoparasitoids differs on cultivated and wild Brassica populations. J. Chem. Ecol., 34: 132-143.

Halitschke, R., Schittko, U., Pohnert, G., Boland, W., Baldwin, I.T. 2001. Molecular interactions between the specialist herbivore Manduca sexta (Lepidoptera, Sphingidae) and its natural host Nicotiana attenuata. III. Fatty acidamino acid conjugates in herbivore oral secretions are necessary and sufficient for herbivore specific plant responses. Plant Physiol., 125: 711-17.

Hammer, K.A., Carson, C.F., and Riley, T.V. 2003. Antifungal activity of the components of Melaleuca alternifolia (tea tree) oil. J. Appl. Microbiol., 95: 853860.

Hatanaka, A., Kajiwara, T., and Sekiya, J. 1987. Biosynthetic pathway For C6-aldehydes formation from linolenic acid in green leaves. Chem. Phys. Lipids, 44: 341-361.

Heil, M. 2009. Damaged-self recognition in plant herbivore defence. Trends Plant Sci., 14: 356-363.

Heil, M. 2014. Herbivore-induced plant volatiles: targets, perception and unanswered questions. New Phytol., 204: 297-306.

Heil, M., 2008. Indirect defence via tritrophic interactions. New Phytol., 178: 41-61.

Hilker, M., and Meiners, T. 2002. Induction of plant responses towards oviposition and feeding of herbivorous arthropods: A comparison. Entomol. Exp. Appl., 104: 181-192.

James, D.G. 2003. Synthetic herbivore-induced plant volatiles as field attractants for beneficial insects. Environ. Entomol., 32: 977-982.

Kant, M.R., Ament, K., Sabelis, M.W., Haring, M.A., and Schuurink, R.C. 2004. Differential timing of spider mite-induced direct and indirect defenses in tomato plants. Plant Physiol., 135: 483-495.

Kessler, A., and Baldwin, I.T. 2001. Defensive function of herbivore induced plant volatile emissions in nature. Sci., 291: 2141- 2144. 
Kessler, A., and Baldwin, I.T. 2002. Plant responses to insect herbivory: the emerging molecular analysis. Аnnu. Rev. Plant Biol., 53: 299-328.

Kessler, A., Halitschke, R., Diezel, C., and Baldwin, I. T. 2006. Priming of plant defense responses in nature by airborne signaling between Artemisia and Nicotiana attenuata. Plant Anim. Interact., 148: 280-292.

Khan, Z.R., AmpongNyarko, K., Chiliswa, P., Hassanali, A., Kimani, S., Lwande, W., Overholt, W.A., Pickett, J.A., Smart, L.E., and Wadhams, L.J. 1997. Intercropping increases parasitism of pests. Nature, 388: 631-632.

Knudsen, J.T., and Gershenzon, J. 2006. The chemistry diversity of floral scent. In: Biol. Floral Scent. pp. 27-52.

Liu, J., Xia, K.F., Zhu, J.C., Deng, Y.G., Huang, X.L., an HuBL, XuX, Xu, Z.F. 2006. The nightshade proteinase inhibitor IIb gene is constitutively expressed in glandular trichomes. Plant Cell Physiol., 47: 1274-1284.

Loreto, F. and Schnitzler, J.P. 2010. Abiotic stresses and induced BVOCs. Trends Plant Sci., 15: 154-166.

Loreto, F., and Velikova, V. 2001. Isoprene produced by leaves protects the photosynthetic apparatus against ozone damage, quenches ozone products, and reduces lipid peroxidation of cellular membranes. Plant Physiol., 127: 17811787.

Loreto, F., Forster, A., Durr, M., Csiky, O., and Seufert, G. 1998. On the monoterpene emission under heat stress and on the increased thermotolerance of leaves of Quercus ilex L. fumigated with selected monoterpenes. Plant Cell Environ., 21: 101-107.

Loreto, F., Mannozzi, M., Maris, C., Nascetti, P., Ferranti, F., and Pasqualini, S. 2001. Ozone quenching properties of isoprene and its antioxidant role in leaves. Plant Physiol., 126: 993-1000.

Loughrin, J., and Manukian, A. 1995. Volatiles emitted by different cotton varieties damaged by feeding beet armyworm larvae. J. Chem. Ecol., 21: 1217-1227.

Mattiacci, L., Dicke, M., and Posthumus, M.A 1995. beta-Glucosidase: an elicitor of herbivore- induced plant odor that attracts host-searching parasitic wasps. Proc. Natl. Acad. Sci. USA. 92: 2036-40.

Mendelsohn, R., Balick, M.J. 1995. The value of undiscovered pharmaceuticals in tropical forests. Econ. Bot. 49: 223-28.

Mithofer, A., and Boland, W. 2008. Recognition of herbivory-associated molecular patterns. Plant Physiol., 146: 825-31.

Mumm, R. and Dicke M. 2010. Variation in natural plant products and the attraction of bodyguards involved in indirect plant defense. Canadian J. Zool. Revue Canadienne De Zoologie. 88: 628-667.

Naoumkina, M.A., Zhao, Q., Gallego-Giraldo, L., Dai, X., Zhao, P.X., and Dixon, R.A. 2010. Genome-wide analysis of phenylpropanoid defence pathways. $\mathrm{Mol}$ Plant Pathol., 11: 829-846.

Pare, P.W., and Tumlinson, J.H. 1997. De novo biosynthesis of volatiles induced by insect herbivory in cotton plants. Plant Physiol., 114: 1161-1167.

Penuelas, J., Llusia, J., Asensio, D., and MunneBosch, S. 2005. Linking isoprene with plant thermotolerance, antioxidants and monoterpene emissions. Plant Cell Environ., 28: 278- 286.

Pieterse, C.M.J., van der Does, D., Zamioudis, C., Leon-Reyes, A., van Wees, S.C.M. 2012. Hormonal modulation of plant immunity. Annu. Rev. Cell Dev. Biol., 28: 489-521.

Rasmann, S., Kollner, T.G., Degenhardt, J., Hiltpold, I., Toepfer, S., Kuhlmann, U., Gershenzon, J., and Turlings, T.C.J. 2005. Recruitment of entomopathogenic nematodes by insect- damaged maize roots. Nature, 434: 732-737.

Rosenkranz, M., Pugh, T.A.M., Schnitzler, J.P. and Arneth, A. 2014. Effect of land-use change and management on BVOC emissions- selecting climate-smart cultivars. J. Experimental Bot., 38: 1896- 
1912.

Ruther, J., and Kleier, S. 2005. Plant-plant signaling: Ethylene synergizes volatile emission in Zea mays induced by exposure to (Z)-3-hexen-1-ol. J. Chem. Ecol., 31: 2217-2222.

Schilmiller, A.L., Last, R.L., and Pichersky, E. 2008. Harnessing plant trichome biochemistry for the production of useful compounds. Plant J., 54: 702-711.

Schoonhoven, L.M., van Loon, J.J.A., and Dicke, M., 2005. Insect-Plant Biology. Oxford University Press, Oxford. Science. 311: 812-815.

Sharkey, T.D., and Yeh, S.S. 2001. Isoprene emission from plants. Annu. Rev. Plant. Phys., 52: 407-436.

Sharkey, T.D., Chen, X.Y., and Yeh, S. 2001. Isoprene increases thermo tolerance of fosmidomycin-fed leaves. Plant Physiol., 125: 2001-2006.

Steppuhn, A., Gase, K., Krock, B., Halitschke, R. and Baldwin, I.T. 2004. Nicotine's defensive function in nature. PLoS Biol., 2: 1074- 1080.

Tholl, D., Chen, F., Petri, J., Gershenzon, J., and Pichersky, E. 2005. Two sesquiterpene synthases are responsible for the complex mixture of sesquiterpenes emitted from Arabidopsis flowers. Plant J., 42: 757-771.
Truitt, C.L.,Wei, H.X., and Pare, P.W. 2004. A plasma membrane protein from Zea mays binds with the herbivore elicitor volicitin. Plant Cell, 16: 523-32.

Turlings, T.C.J., Tumlinson, J.H., and Lewis, W.J. 1990. Exploitation of herbivoreinduced plant odors by host-seeking parasitic wasps. Sci., 250: 1251-1253.

Vancanneyt, G., Sanz, C., Farmaki, T., Paneque, M., Ortego, F., Castanera, P., and Sanchez- Serrano, J.J. 2001. Hydroperoxide lyase depletion in transgenic potato plants leads to an increase in aphid performance. Proc. Natl. Acad. Sci. USA, 98: 8139-8144.

Vet, L.E.M., and Dicke, M. 1992. Ecology of infochemical use by natural enemies in a tritrophic context. Annu. Rev. Entomol., 37: 141-172.

Walling, L.L. 2000. The myriad plant responses to herbivores. J. Plant Growth Regul., 19: 195- 216.

Wink, M. 2003 Evolution of secondary metabolites from an ecological and molecular phylogenetic perspective. Phytochem., 64: 3-19.

Yu, H., Kowalski, S.P., and Steffens, J.C. 1992. Comparison of polyphenol oxidase expression in glandular trichomes of Solanum and Lycopersicon species. Plant Physiol., 100: 1885- 1890.

\section{How to cite this article:}

Rahul Kumar Meena, Sumit Jangra, Zeenat Wadhwa, Monika and Leela Wati. 2017. Role of Plant Volatiles in Defense and Communication. Int.J.Curr.Microbiol.App.Sci. 6(4): 300-313. doi: https://doi.org/10.20546/ijcmas.2017.604.033 